\title{
On the cardinality of power homogeneous Hausdorff spaces
}

\author{
by
}

\author{
G. J. Ridderbos (Amsterdam)
}

\begin{abstract}
We prove that the cardinality of power homogeneous Hausdorff spaces $X$ is bounded by $d(X)^{\pi \chi(X)}$. This inequality improves many known results and it also solves a question by J. van Mill. We further introduce $\Delta$-power homogeneity, which leads to a new proof of van Douwen's theorem.
\end{abstract}

1. Introduction. A space $X$ is homogeneous if for every $x, y \in X$ there is a homeomorphism $h$ of $X$ such that $h(x)=y$. A space $X$ is called power homogeneous if $X^{\mu}$ is homogeneous for some cardinal number $\mu$. By $\pi \chi(X)$ and $\pi w(X)$ we denote the $\pi$-character and $\pi$-weight respectively. By $d(X), w(X), \psi w(X)$ and $c(X)$ we denote density, weight, pseudo-weight and cellularity.

In 1978, E. van Douwen proved in [4] that the cardinality of power homogeneous Hausdorff spaces $X$ is bounded by $2^{\pi w(X)}$. Applying results of Shapirovskiı [12] and Ismail [6], A. V. Arkhangel'skiǔ noted in [1, Theorem 1.5] that the cardinality of homogeneous regular spaces $X$ is bounded by $2^{\pi \chi(X) c(X)}$. Since always $\pi \chi(X) c(X) \leq \pi w(X)$ and strict inequality is possible, this result improves van Douwen's theorem for the class of homogeneous spaces. Recently, J. van Mill [8] extended Arkhangel'skii's result to the class of power homogeneous compacta. In his paper van Mill asks whether this result can also be proved for power homogeneous spaces without the assumption of compactness (see [8, Remark 2.7]). A partial answer to this question was provided by A. Bella in [3]. In the present paper we provide a full positive answer to van Mill's question (see Corollary 3.5).

In [4] van Douwen studies power homogeneous spaces by looking at the number of possible ways certain sequences of open sets cluster at points. This same method was applied by van Mill in [8]. In the present paper we introduce an entirely different technique which follows from results in [2].

2000 Mathematics Subject Classification: 54A25, 54B10.

Key words and phrases: power homogeneity, density, $\pi$-character, cellularity. 
This new technique has a number of interesting applications. First of all, we prove that the cardinality of power homogeneous spaces $X$ is bounded by $d(X)^{\pi \chi(X)}$, which improves van Douwen's theorem. This bound surfaced earlier. By the method of proof used by Ismail in [6], some mathematicians have noted that the cardinality of a homogeneous space $X$ is bounded by $d(X)^{\pi \chi(X)}$ (see for example [13, Theorem 1.14] and [9, Proposition 2.2.7]). By results of van Mill in [8] one can easily show that this bound is also valid for power homogeneous compacta (cf. Proposition 2.3).

The concept of a power homogeneous space is rather new. A close look at the results in the literature shows that often not all available properties of a homogeneous power space are used. This leads to a notion which is introduced in Section 2 and which we call $\Delta$-power homogeneity. Every power homogeneous space is also $\Delta$-power homogeneous. In Section 4 we prove a reflection theorem for this property and this leads to a very simple and elegant proof of van Douwen's theorem. The simplicity lies in the fact that in our proof we only look at "small" powers of the space under consideration.

In the final section of this paper we look at the connection between $\Delta$-power homogeneity and power homogeneity. We do not know whether these notions are equivalent.

2. Preliminaries. All spaces are assumed to be Hausdorff. All product spaces in this paper carry the usual product topology. Whenever $\left\{X_{i}: i \in I\right\}$ is a collection of topological spaces and $Y=\prod\left\{X_{i}: i \in I\right\}$ is the product space, then for $A \subseteq I$ by $Y_{A}$ we denote the product $\prod\left\{X_{i}: i \in A\right\}$. By $\pi_{A}$ we denote the natural projection of $Y$ onto $Y_{A}$. If $B \subseteq A \subseteq I$, then by $\pi_{A \rightarrow B}$ we denote the projection from $Y_{A}$ onto $Y_{B}$. If $i \in I$, then we write $\pi_{i}$ instead of $\pi_{\{i\}}$. If $y \in Y$ then by $y_{A}$ we denote the point $\pi_{A}(y)$. If $Z \subseteq Y$, then $Z_{A}=\pi_{A}[Z]$.

Many of the product spaces that we deal with in this paper are of the form $X^{\mu}$ for some infinite cardinal number $\mu$. In that case we write $X^{A}$ for $\left(X^{\mu}\right)_{A}$. Furthermore, by $\pi$ we denote $\pi_{0}$, the projection on the first co-ordinate.

Whenever $x \in X$, by $\underline{x}$ we denote the element of $X^{\mu}$ which is equal to $x$ on all co-ordinates. By $\Delta(X, \mu)$ we denote the diagonal in $X^{\mu}$, thus $\Delta(X, \mu)=\{\underline{x}: x \in X\}$. We will call a space $X^{A} \Delta$-homogeneous if for all points $x, z \in \Delta(X, A)$ there is a homeomorphism of $X^{A}$ mapping $x$ onto $z$. We will call a space $\Delta$-power homogeneous if for some cardinal number $\mu$, the space $X^{\mu}$ is $\Delta$-homogeneous. Clearly, every power homogeneous space is $\Delta$-power homogeneous. Furthermore, if $X^{\mu}$ is $\Delta$-homogeneous and $\lambda \geq \mu$ then $X^{\lambda}$ is $\Delta$-homogeneous.

The set of all autohomeomorphisms of a space $X$ is denoted by $\operatorname{Aut}(X)$ and we let $\operatorname{tpe}(x, X)=\{h(x): h \in \operatorname{Aut}(X)\}$ be the type of $x$ in $X$. 
As a warm-up exercise we prove the following proposition, from which it follows that the cardinality of homogeneous spaces $X$ is bounded by $d(X)^{\pi \chi(X)}$. This was noted independently by de la Vega [13, Theorem 1.14] and the author [9, Proposition 2.2.7]. Its simple proof plays a major part in the proof of Theorem 3.4.

Proposition 2.1. Suppose $A$ is some type in $X$. Then $|A| \leq d(X)^{\pi \chi(X)}$.

Proof. Let $A=\operatorname{tpe}(x, X)$. For every $y \in A$ we may fix a homeomorphism $h_{y}: X \rightarrow X$ such that $h_{y}(x)=y$. We fix a dense set $D$ in $X$ with $|D|=d(X)$ and a local $\pi$-base $\mathcal{U}$ at $x$ in $X$ with $|\mathcal{U}| \leq \pi \chi(X)$.

We define a map $H: A \rightarrow D^{\mathcal{U}}$ as follows. Fix some well-ordering on $D$, and for $y \in A$ and $U \in \mathcal{U}$ let

$$
H(y)(U)=\min \left\{d \in D: d \in h_{y}[U]\right\} .
$$

Since $D$ is dense this is well-defined. We leave it to the reader to verify that $H$ is injective. This is similar to the second half of the proof of Theorem 3.4 .

The following result was proved in [2, Corollary 2.3]. In Section 3 we will apply this result for the special case when $Y=X^{\mu}, I=\mu$ and $i=0$.

Proposition 2.2. Let $Y=\prod\left\{X_{i}: i \in I\right\}$ and suppose that $h: Y \rightarrow Y$ is a homeomorphism. Suppose further that for some $i \in I, \pi \chi\left(X_{i}\right) \leq \kappa$. Let $Z$ be a subset of $Y$ with $|Z| \leq \kappa$. Then there is a set of co-ordinates $A \in[I] \leq \kappa$ such that for all $y \in Z$,

$$
h \pi_{A}^{-1}\left(y_{A}\right) \subseteq \pi_{i}^{-1}\left(h(y)_{i}\right) .
$$

The following theorem follows from Theorem 3.4 below. For completeness' sake we show here that it also follows from van Mill's inequality in $[8]$.

Proposition 2.3. If $X$ is compact and power homogeneous, then $|X| \leq$ $d(X)^{\pi \chi(X)}$.

Proof. Since $X$ is compact, $w(X)=\psi w(X)$ (cf. [7, 3.11]). Since $X$ is also power homogeneous, it follows that $|X| \leq w(X)^{\pi \chi(X)}$ (see [8, Theorem $2.5])$. So it suffices to show that $\psi w(X) \leq d(X)^{\pi \chi(X)}$.

If $D$ is any dense set and $\pi \chi(X)=\kappa$, then it is easily verified that the collection given by

$$
\left\{X \backslash \bar{E}: E \in[D]^{\leq \kappa}\right\}
$$

is a pseudo-base for $X$. Since $\left|[D]^{\leq \kappa}\right|=|D|^{\kappa}$, it follows that $\psi w(X) \leq$ $d(X)^{\pi \chi(X)}$. 
3. The cardinality of power homogeneous Hausdorff spaces. In this section we fix some space $X$, a cardinal number $\mu$ and a point $p \in$ $\Delta(X, \mu)$. We further assume that $\mathcal{U}$ is a local $\pi$-base at $\pi(p)$ in $X$. Whenever $A \subseteq \mu$, then by $\mathcal{U}(A)$ we denote the collection

$$
\left\{\pi_{A \rightarrow B}^{-1}\left[\prod_{b \in B} U_{b}\right]: B \in[A]^{<\omega}, \forall b \in B\left(U_{b} \in \mathcal{U}\right)\right\} .
$$

Then $\mathcal{U}(A)$ is a local $\pi$-base at $p_{A}$ in $X^{A}$ and $|\mathcal{U}(A)| \leq|A| \cdot|\mathcal{U}|$. Furthermore if $A=\bigcup_{n<\omega} A_{n}$ where $\left\{A_{n}: n<\omega\right\}$ is some increasing sequence of subsets of $\mu$, then

$$
\mathcal{U}(A)=\bigcup_{n<\omega} \pi_{A \rightarrow A_{n}}^{-1} \mathcal{U}\left(A_{n}\right),
$$

where $\pi_{A \rightarrow B}^{-1} \mathcal{U}(B)$ denotes the collection $\left\{\pi_{A \rightarrow B}^{-1}[U]: U \in \mathcal{U}(B)\right\}$. The following theorem follows from Theorem 2.2 and a recursive construction.

Theorem 3.1. Let $D$ be a dense subset of $X$, and $h: X^{\mu} \rightarrow X^{\mu} a$ homeomorphism. If $\pi \chi(X) \leq \kappa,|\mathcal{U}| \leq \kappa$ and $B \in[\mu]^{\leq \kappa}$, then there is a set $A \in[\mu]^{\leq \kappa}$ such that $B \subseteq A$ and for all $U \in \mathcal{U}(A)$ there is some $e \in X^{\mu}$ satisfying:

(1) $\pi h(e)=d \in D$ and $e \in \pi_{A}^{-1}[U]$,

(2) $h \pi_{A}^{-1}\left(e_{A}\right)$ is contained in $\pi^{-1}(d)$.

Proof. We may construct an increasing sequence $\left\{A_{n}: n<\omega\right\} \subseteq[\mu] \leq \kappa$ where $A_{0}=B$, such that for all $U \in \mathcal{U}\left(A_{n}\right)$ there is some $e \in X^{\mu}$ satisfying

(1) $\pi h(e)=d \in D$ and $e \in \pi_{A_{n}}^{-1}[U]$,

(2) $h \pi_{A_{n+1}}^{-1}\left(e_{A_{n+1}}\right)$ is contained in $\pi^{-1}(d)$.

For (1), this follows from the fact that $D$ is dense in $X$, so that $\pi h \pi_{A_{n}}^{-1}[U]$ $\cap D \neq \emptyset$. For (2), we just apply Theorem 2.2 ; given $A_{n}$ we may find $A_{n+1}$ such that $(2)$ is satisfied since $\left|\mathcal{U}\left(A_{n}\right)\right| \leq \kappa$.

We set $A=\bigcup_{n<\omega} A_{n}$. Then the conditions in the theorem are satisfied since $\mathcal{U}(A)=\bigcup_{n<\omega} \pi_{A \rightarrow A_{n+1}}^{-1} \mathcal{U}\left(A_{n+1}\right)$.

Whenever $A$ and $B$ are subsets of $\mu$ such that $|A|=|B|$ and $|\mu \backslash A|=$ $|\mu \backslash B|$, then there is a natural homeomorphism of $X^{\mu}$ that realizes a coordinate change from $A$ to $B$. We denote this homeomorphism by $g_{A \rightarrow B}$. It is defined as follows. Let $g: \mu \rightarrow \mu$ be a bijection such that $g[A]=B$. Then $g_{A \rightarrow B}: X^{\mu} \rightarrow X^{\mu}$ is defined co-ordinatewise for $\beta<\mu$ :

$$
g_{A \rightarrow B}(x)_{\beta}=x_{\alpha} \stackrel{\text { def }}{\Longleftrightarrow} g(\alpha)=\beta .
$$

The following is a simple lemma concerning the homeomorphism $g_{A \rightarrow B}$. 
Lemma 3.2. Suppose $A$ and $B$ are subsets of $\mu$ with $|A|=|B|$ and $|\mu \backslash A|=|\mu \backslash B|$. Then

(1) $\left(g_{A \rightarrow B}\left[\pi_{A}^{-1}[U]\right]\right)_{B} \in \mathcal{U}(B)$ if and only if $U \in \mathcal{U}(A)$,

(2) for all $Y \subseteq X^{\mu}$,

$$
g_{A \rightarrow B}\left[\pi_{A}^{-1}\left[Y_{A}\right]\right]=\pi_{B}^{-1}\left[\left(g_{A \rightarrow B}[Y]\right)_{B}\right] .
$$

Using a co-ordinate change, we can control the set $A$ which is provided by Theorem 3.1. We make this precise in the following corollary.

Corollary 3.3. Let $\pi \chi(X) \leq \kappa,|\mathcal{U}| \leq \kappa$ and let $\mu \geq \kappa$. Suppose that $X^{\mu}$ is $\Delta$-homogeneous and $D$ is a dense subset of $X$. Then for every $q \in$ $\Delta(X, \mu)$ there is a homeomorphism $h_{q}: X^{\mu} \rightarrow X^{\mu}$ satisfying the following conditions:

(1) $h_{q}(p)=q$,

(2) for all $U \in \mathcal{U}(\kappa)$ there is some $e \in X^{\mu}$ satisfying:

(a) $\pi h_{q}(e)=d \in D$ and $e \in \pi_{\kappa}^{-1}[U]$,

(b) $h_{q} \pi_{\kappa}^{-1}\left(e_{\kappa}\right)$ is contained in $\pi^{-1}(d)$.

Proof. Since $X^{\mu}$ is $\Delta$-homogeneous, there is a homeomorphism $h$ of $X^{\mu}$ with $h(p)=q$. We apply Theorem 3.1 with $B=\kappa$ to obtain $A \in[\mu] \leq \kappa$ with the given properties. Since $B \subseteq A$, we have $|A|=\kappa$. There are two cases to consider. First of all, if $\kappa=\mu$, then $A=\kappa$ and conditions (2a) and (2b) are valid for $h$.

Next assume that $\kappa<\mu$. Then $|A|=\kappa$ and $|\mu \backslash A|=\mu=|\mu \backslash \kappa|$. Therefore we may apply the co-ordinate change $g_{\kappa \rightarrow A}$. We let $h_{q}=h \circ g_{\kappa \rightarrow A}$. Since $g_{\kappa \rightarrow A}(p)=p$, we have $h_{q}(p)=q$. By Lemma 3.2 conditions $(2 \mathrm{a})$ and $(2 \mathrm{~b})$ are valid for $h_{q}$ since they are valid for $h$ when $\kappa$ is replaced by $A$.

Theorem 3.4. Suppose $X$ is $\Delta$-power homogeneous. Then $|X| \leq$ $d(X)^{\pi \chi(X)}$.

Proof. Let $\kappa=\pi \chi(X)$. We may assume that $X^{\mu}$ is $\Delta$-homogeneous where $\mu \geq \kappa$. Let $D$ be some dense subset of $X$ with $|D|=d(X)$. For every $q \in \Delta(X, \mu)$ we fix a homeomorphism $h_{q}$ as in the previous corollary. Since $\pi \chi(X)=\kappa$, we may assume that the size of the local $\pi$-base $\mathcal{U}$ is equal to $\kappa$. Then $\mathcal{U}(\kappa)$ is a local $\pi$-base at $p_{\kappa}$ in $X^{\kappa}$ of size equal to $\kappa$.

Fix some well-ordering of $X^{\mu}$. We define a map $H: \Delta(X, \mu) \rightarrow D^{\mathcal{U}(\kappa)}$ as follows. Whenever $q \in \Delta(X, \mu)$ and $U \in \mathcal{U}(\kappa)$ then $H(q)(U)=\pi h_{q}(e)$ where

$$
e=\min \left\{x \in X^{\mu}: x \text { satisfies conditions (2a) and (2b) of Corollary 3.3 }\right\} .
$$

Note that in particular $H(q)(U) \in D$ so $H$ is well-defined. We will show that $H$ is injective, which will complete the proof. So suppose $q, r \in \Delta(X, \mu)$ 
where $q \neq r$. In $X$, we may fix disjoint open neighbourhoods $V_{q}$ and $V_{r}$ of $\pi(q)$ and $\pi(r)$ respectively. The set $V$ given by

$$
\pi_{\kappa} h_{q}^{-1} \pi^{-1}\left[V_{q}\right] \cap \pi_{\kappa} h_{r}^{-1} \pi^{-1}\left[V_{r}\right]
$$

is an open neighbourhood of $p_{\kappa}$ in $X^{\kappa}$. Since $\mathcal{U}(\kappa)$ is a local $\pi$-base at $p_{\kappa}$, there is some $U \in \mathcal{U}(\kappa)$ which is contained in the neighbourhood $V$. We will prove the following claim,

Claim. $H(q)(U) \in V_{q}$ and $H(r)(U) \in V_{r}$.

Proof of Claim. We prove the statement only for $q$, the case for $r$ is identical. So suppose that $H(q)(U)=\pi h_{q}(e)=d$. Then $e \in \pi_{\kappa}^{-1}[U]$, so $e_{\kappa} \in U$. Since $U \subseteq V \subseteq \pi_{\kappa} h_{q}^{-1} \pi^{-1}\left[V_{q}\right]$ it follows that

$$
\pi_{\kappa}^{-1}\left(e_{\kappa}\right) \cap h_{q}^{-1} \pi^{-1}\left[V_{q}\right] \neq \emptyset,
$$

and by applying $h_{q}$ we have

$$
h_{q} \pi_{\kappa}^{-1}\left(e_{\kappa}\right) \cap \pi^{-1}\left[V_{q}\right] \neq \emptyset .
$$

Since $h_{q} \pi_{\kappa}^{-1}\left(e_{\kappa}\right) \subseteq \pi^{-1}(d)$, it follows that $d \in V_{q}$ and this proves the Claim.

Since $V_{q} \cap V_{r}=\emptyset$ it follows from the claim that $H(q)(U) \neq H(r)(U)$, and thus $H(q) \neq H(r)$. We have shown that $H$ is injective and therefore $|X|=|\Delta(X, \mu)| \leq|D|^{|\mathcal{U}(\kappa)|}=d(X)^{\pi \chi(X)}$.

Since every power homogeneous space is also $\Delta$-power homogeneous, it follows that the cardinality of power homogeneous spaces is bounded by $d(X)^{\pi \chi(X)}$. By the same observation, the following corollary answers van Mill's question in [8].

Corollary 3.5. If $X$ is a $\Delta$-power homogeneous regular space, then $|X| \leq 2^{\pi \chi(X) c(X)}$.

Proof. The assertion follows from Theorem 3.4 and the fact that $d(X) \leq$ $\pi \chi(X)^{c(X)}$, which was proved by Shapirovskiu in [12, Theorem 3].

By $t(X)$ and $\operatorname{pct}(X)$ we denote the tightness and pointwise compactness type respectively; see Sections 1 and 4 in [2] for the respective definitions. By $L(X)$ we denote the Lindelöf degree. The following corollary answers Question 4.10 in [2].

Corollary 3.6. If $X$ is a power homogeneous regular space, then

$$
|X| \leq 2^{t(X) \operatorname{pct}(X) L(X)} .
$$

Proof. Since $X$ is a power homogeneous regular space, we have $d(X) \leq$ $2^{t(X) \operatorname{pct}(X) L(X)}$ by [2, Corollary 4.5]. Furthermore, $\pi \chi(X) \leq t(X) \operatorname{pct}(X)$ by [13, Corollary 1.13]. So Theorem 3.4 implies that $|X| \leq 2^{t(X) \operatorname{pct}(X) L(X)}$. 
4. $\Delta$-power homogeneity reflects to small powers. As an application of the results obtained in this section, we present a new proof of van Douwen's theorem without using his "clustering" method. This method is very powerful since one can prove cardinality results about a space $X$ by looking at some power $X^{\mu}$, regardless of the size of the cardinal number $\mu$. The main result in this section is that if $X$ is $\Delta$-power homogeneous and $\pi w(X)=\kappa$, then $X^{\kappa}$ is $\Delta$-homogeneous. We are then able to prove van Douwen's theorem by just looking at the space $X^{\kappa}$.

The following result is similar to [11, Theorem 4], see also [5, Exercise 2.7.12]. It can also be obtained as an application of Theorem 2.2. Recall from [11, Theorem 3] that if $Y=\prod\left\{X_{i}: i \in I\right\}$ and $d\left(X_{i}\right) \leq \kappa$ for all $i \in I$, then the closure of an open set $U$ in $Y$ depends on not more than $\kappa$ co-ordinates, which means that $\bar{U}=\pi_{A}^{-1}\left[\pi_{A}[\bar{U}]\right]$ for some $A \in[I] \leq \kappa$.

Theorem 4.1. Let $Y=\prod\left\{X_{i}: i \in I\right\}$ and suppose that $h: Y \rightarrow Y$ is a homeomorphism. Suppose further that $d\left(X_{i}\right) \leq \kappa$ for all $i \in I$, and $\pi w\left(X_{j}\right) \leq \kappa$ for some $j \in I$. Then there is a set $A \in[I]^{\leq \kappa}$ such that for all $w, z \in Y$,

$$
w_{A}=z_{A} \Rightarrow h(w)_{j}=h(z)_{j} .
$$

Proof. Fix a $\pi$-base $\mathcal{U}$ in $X_{j}$ of size $\leq \kappa$. Then we may fix a set of co-ordinates $A \in[I]^{\leq \kappa}$ such that for every $U \in \mathcal{U}$, the closure of $h^{-1} \pi_{j}^{-1}[U]$ depends on the co-ordinates in $A$. We will show that $A$ is as required.

So let $w, z \in Y$ with $w_{A}=z_{A}$ and suppose $p=h(w)_{j} \neq h(z)_{j}=q$. Then we may fix a neighbourhood $V$ of $p$ in $X_{j}$ with $q \notin \bar{V}$. Let $\mathcal{V}=\{U \in \mathcal{U}$ : $U \subseteq V\}$. Since $\mathcal{U}$ is a $\pi$-base in $X_{j}$ we have $p \in \operatorname{Cl} \cup \mathcal{V}$. But then $w \in \bar{F}$ where

$$
F=\bigcup\left\{\mathrm{Cl} h^{-1} \pi_{j}^{-1}[U]: U \in \mathcal{V}\right\} .
$$

By construction we have $F=\pi_{A}^{-1}\left[\pi_{A}[F]\right]$, so also $\bar{F}=\pi_{A}^{-1}\left[\pi_{A}[\bar{F}]\right]$. Since $z_{A}=w_{A} \in \pi_{A}[\bar{F}]$, it follows that $z \in \pi_{A}^{-1}\left[\pi_{A}[\bar{F}]\right]=\bar{F}$. But we also have $\bar{F} \subseteq h^{-1} \pi_{j}^{-1}[\bar{V}]$, and therefore it follows that $q=h(z)_{j} \in \bar{V}$, which is impossible.

Corollary 4.2. Let $h: X^{\mu} \rightarrow X^{\mu}$ be a homeomorphism and suppose $\pi w(X) \leq \kappa$. If $B \in[\mu]^{\leq \kappa}$ then there is a set $A \in[\mu]^{\leq \kappa}$ such that for all $w, z \in X^{\mu}$,

$$
w_{A}=z_{A} \Rightarrow h(w)_{B}=h(z)_{B} .
$$

Proof. We may view $X^{\mu}$ as the product space of $X^{B}$ and $X_{\alpha}$ for $\alpha \in$ $\mu \backslash B$. Since $\pi w\left(X^{B}\right)=\pi w(X) \cdot|B| \leq \kappa$ (cf. [7, 5.3]), the statement follows from Theorem 4.1. 
Note that in the previous corollary, any set containing the set $A$ also satisfies the conclusion. In particular, the set $A$ may be taken so that it contains $B$.

Theorem 4.3. Let $h: X^{\mu} \rightarrow X^{\mu}$ be a homeomorphism and let $\pi w(X) \leq \kappa$. Suppose $B \in[\mu]^{\leq \kappa}$. Then there is a set $A \in[\mu]^{\leq \kappa}$ such that $B \subseteq A$ and for all $w, z \in X^{\mu}$,

$$
w_{A}=z_{A} \Leftrightarrow h(w)_{A}=h(z)_{A} .
$$

Proof. By Corollary 4.2 we may construct a sequence $\left(A_{n}\right)_{n}$ which satisfies the following conditions for all $n<\omega$ :

(1) $A_{0}=B, A_{n} \in[\mu] \leq \kappa$ and $A_{n} \subseteq A_{n+1}$.

(2) For all $w, z \in X^{\mu}, w_{A_{2 n+1}}=z_{A_{2 n+1}} \Rightarrow h(w)_{A_{2 n}}=h(z)_{A_{2 n}}$,

(3) For all $w, z \in X^{\mu}, h(w)_{A_{2 n+2}}=h(z)_{A_{2 n+2}} \Rightarrow w_{A_{2 n+1}}=z_{A_{2 n+1}}$.

Now set $A=\bigcup_{n<\omega} A_{n}$ and the theorem follows.

Note that if $A$ is as in the previous theorem, then it is also the case that for all $w, z \in X^{\mu}$,

$$
w_{A}=z_{A} \Leftrightarrow h^{-1}(w)_{A}=h^{-1}(z)_{A} .
$$

THEOREM 4.4. Let $X$ be a topological space and suppose $\pi w(X) \leq \kappa \leq \mu$. Suppose further that $h: X^{\mu} \rightarrow X^{\mu}$ is a homeomorphism. If $B \subseteq \mu$ with $|B| \leq \kappa$ then there is a subset $A \subseteq \mu$ with $|A| \leq \kappa$ such that $B \subseteq A$ and $A$ has the following property: if $i: X^{A} \rightarrow X^{\mu}$ is any continuous injection with $\pi_{A} \circ i=\mathrm{id}_{X^{A}}$ then

$$
\pi_{A} \circ h \circ i: X^{A} \rightarrow X^{A}
$$

is a homeomorphism.

Proof. By Theorem 4.3 we may choose $A \subseteq \mu$ such that $B \subseteq A,|A| \leq \kappa$ and $A$ has the following property: for all $x, y \in X^{\mu}$,

$$
x_{A}=y_{A} \Leftrightarrow h(x)_{A}=h(y)_{A} \quad \text { and } \quad x_{A}=y_{A} \Leftrightarrow h^{-1}(x)_{A}=h^{-1}(y)_{A} .
$$

Let $i: X^{A} \rightarrow X^{\mu}$ be any continuous injection such that $\pi_{A} \circ i=\operatorname{id}_{A}$ and let $f=\pi_{A} \circ h \circ i$ and $g=\pi_{A} \circ h^{-1} \circ i$. Then, clearly, $f$ and $g$ are continuous.

We will show that $f \circ g=g \circ f=\operatorname{id}_{X^{A}}$. So let $z \in X^{A}$. Then by definition of $g$ we have

$$
i(g(z))_{A}=g(z)=h^{-1} i(z)_{A} .
$$

By $(*)$ it follows that

$$
f(g(z))=h i(g(z))_{A}=i(z)_{A}=z .
$$

Similarly it follows that $g(f(z))=z$ for all $z \in X^{A}$. This proves the theorem. 
TheOREM 4.5. Suppose $X$ is $\Delta$-power homogeneous and $\pi w(X)=\kappa$. Then $X^{\kappa}$ is $\Delta$-homogeneous.

Proof. We may choose $\mu \geq \kappa$ such that $X^{\mu}$ is $\Delta$-homogeneous. Instead of choosing arbitrary elements of $\Delta(X, \kappa)$, we choose $x, y \in \Delta(X, \mu)$ arbitrarily and show that for some homeomorphism $g$ of $X^{\kappa}, g\left(x_{\kappa}\right)=y_{\kappa}$.

Since $X^{\mu}$ is $\Delta$-homogeneous, we may fix a homeomorphism $h: X^{\mu} \rightarrow X^{\mu}$ with $h(x)=y$. Let $A \in[\mu]^{\kappa}$ be as in the previous theorem. Let $i: X^{A} \rightarrow X^{\mu}$ be the injection given by $i(z)=w$ where $w_{A}=z_{A}$ and $w_{\alpha}=x_{\alpha}$ if $\alpha \in \mu \backslash A$. Then $i\left(x_{A}\right)=x$ and $\pi_{A} \circ i=\operatorname{id}_{X^{A}}$. Let $f=\pi_{A} \circ h \circ i$. Then $f: X^{A} \rightarrow X^{A}$ is a homeomorphism with $f\left(x_{A}\right)=y_{A}$. By a suitable change of co-ordinates we obtain a homeomorphism $g$ of $X^{\kappa}$ which maps $x_{\kappa}$ onto $y_{\kappa}$. It is essential here that both $x$ and $y$ are constant as functions from $\mu$ into $X$.

We now obtain the announced proof of van Douwen's theorem (cf. [4, Theorem 1]). Of course, this result also follows from Theorem 3.4; here we use Proposition 2.1 and Theorem 4.5.

TheOREM 4.6. If $X$ is $\Delta$-power homogeneous then $|X| \leq 2^{\pi w(X)}$.

Proof. Let $\kappa=\pi w(X)$. By Theorem 4.5 it follows that $X^{\kappa}$ is $\Delta$-homogeneous. Therefore the diagonal $\Delta(X, \kappa)$ is contained in some type of $X^{\kappa}$. Since $\pi w\left(X^{\kappa}\right)=\kappa$ (cf. $\left.[7,5.3]\right)$ we have $d\left(X^{\kappa}\right) \leq \kappa$ and $\pi \chi\left(X^{\kappa}\right) \leq \kappa$. It follows from Proposition 2.1 that

$$
|X|=|\Delta(X, \kappa)| \leq \kappa^{\kappa}=2^{\kappa}
$$

5. Power homogeneity and $\Delta$-power homogeneity. Most of the cardinality results in this paper are proved for $\Delta$-power homogeneous spaces. On close inspection of the proofs in [2], it becomes clear that the results there can also be proved for $\Delta$-power homogeneous spaces (see in particular the proof of [2, Corollary 2.9]). Thus if $X$ is a $\Delta$-power homogeneous compact space, then $|X| \leq 2^{t(X)}$, and more generally: if $X$ is a $\Delta$-power homogeneous regular space, then $|X| \leq 2^{t(X) \operatorname{pct}(X) L(X)}$ (see also Corollary 3.6). This raises the question whether $\Delta$-power homogeneity and power homogeneity are equivalent notions. We do not know of an example of a $\Delta$-power homogeneous space which is not power homogeneous.

QUESTION 5.1. Is there a space which is $\Delta$-power homogeneous but which is not power homogeneous?

We have some simple partial results for answering this question. Recall from [2] that the homogeneity index of $X$, $\operatorname{hind}(X)$, is defined as the number of different types in $X$. Thus $\operatorname{hind}(X)=|\{\operatorname{tpe}(x, X): x \in X\}|$. In particular, $\operatorname{hind}(X)$ may be finite. 
Proposition 5.2. Suppose $X^{\mu}$ is $\Delta$-homogeneous and $\operatorname{hind}(X)<\omega$. Then $X^{\mu}$ is homogeneous.

Proof. Let $\operatorname{hind}(X)=n$. We may choose $x_{1}, \ldots, x_{n} \in X$ such that $X=\bigcup\left\{\operatorname{tpe}\left(x_{i}, X\right): 1 \leq i \leq n\right\}$. Let $y$ be the point in $\Delta(X, \mu)$ with all coordinates equal to $x_{1}$. It suffices to show that whenever $z \in\left\{x_{1}, \ldots, x_{n}\right\}^{\mu}$ then $z \in \operatorname{tpe}\left(y, X^{\mu}\right)$.

Claim. Whenever $I \subseteq\{1, \ldots, n\}$ and $z \in\left\{x_{i}: i \in I\right\}^{\mu}$ then $z \in$ $\operatorname{tpe}\left(y, X^{\mu}\right)$.

Proof of Claim. The proof is by induction on $|I|$. If $|I|=1$, then the conclusion follows from $\Delta$-homogeneity of $X^{\mu}$. Next suppose that for all $I \subseteq\{1, \ldots, n\}$ with $|I|=k$ the statement is true and choose $J \subseteq\{1, \ldots, n\}$ with $|J|=k+1$.

Let $z \in\left\{x_{j}: j \in J\right\}^{\mu}$. Then for some $j_{0} \in J$, the set $A=\{\alpha<\mu$ : $\left.z_{\alpha}=x_{j_{0}}\right\}$ is of cardinality $\mu$. Let $j_{1} \in J$ with $j_{1} \neq j_{0}$. By $\Delta$-homogeneity we may pick a homeomorphism $h: X^{A} \rightarrow X^{A}$ such that $h\left(z_{A}\right)_{\alpha}=x_{j_{1}}$ for all $\alpha \in A$. If $B=\mu \backslash A$ and $g$ is the identity on $X^{B}$ then $f=h \times g$ is a homeomorphism of $X^{\mu}$ and $f(z) \in\left\{x_{i}: i \in I\right\}^{\mu}$ where $I=J \backslash\left\{j_{0}\right\}$. Since $|I|=k$ it follows from the induction hypothesis that $f(z) \in \operatorname{tpe}\left(y, X^{\mu}\right)$ and thus $z \in \operatorname{tpe}\left(y, X^{\mu}\right)$.

The proposition follows from the Claim if we take $I=\{1, \ldots, n\}$.

Corollary 5.3. Suppose $\operatorname{hind}(X)<\omega$. Then $X$ is $\Delta$-power homogeneous if and only if $X$ is power homogeneous.

In view of Theorem 4.5 and Question 5.1 one may ask the following question. If $X$ is power homogeneous and $\pi w(X)=\kappa$, is then $X^{\kappa}$ homogeneous? The following theorem provides a positive answer in some special cases.

TheORem 5.4. Suppose $X$ is power homogeneous. Let $\kappa$ be a cardinal number such that $\pi w(X) \leq \kappa$ and $\operatorname{hind}(X)<\operatorname{cf}(\kappa)$. Then $X^{\kappa}$ is homogeneous.

Proof. We may fix a cardinal $\mu \geq \kappa$ such that $X^{\mu}$ is homogeneous. We fix a point $p \in \Delta(X, \mu)$. We will show that for every $x \in X^{\kappa}$ we have $x \in \operatorname{tpe}\left(p_{\kappa}, X^{\kappa}\right)$. Let $\nu=\operatorname{hind}(X)$. We may fix a set $Q \in[X]^{\leq \nu}$ such that $X=\bigcup\{\operatorname{tpe}(q, X): q \in Q\}$. It suffices to show that for every $x \in Q^{\kappa}$, we have $x \in \operatorname{tpe}\left(p_{\kappa}, X^{\kappa}\right)$.

So let $x \in Q^{\kappa}$ be fixed. For every $q \in Q$ we define $A(q)$ as follows:

$$
A(q)=\left\{\alpha \in \kappa: x_{\alpha}=q\right\} .
$$


Note that $\kappa=\bigcup_{q \in Q} A(q)$. So as $|Q|=\nu<\operatorname{cf}(\kappa)$, it follows that $\left|A\left(q^{\prime}\right)\right|=\kappa$ for some $q^{\prime} \in Q$. We define a point $w \in X^{\mu}$ that extends $x$ as follows:

$$
w_{\alpha}= \begin{cases}x_{\alpha} & \text { if } \alpha<\kappa, \\ q^{\prime} & \text { if } \alpha \geq \kappa .\end{cases}
$$

Since $X^{\mu}$ is homogeneous, we may fix a homeomorphism $h: X^{\mu} \rightarrow X^{\mu}$ such that $h(p)=w$. Since $\pi w(X) \leq \kappa$, we may find a set $A \subseteq \mu$ with $\kappa \subseteq A$ and $|A|=\kappa$ as in Theorem 4.4. We let $i: X^{A} \rightarrow X^{\mu}$ be the injection defined for $y \in X^{A}$ by

$$
i(y)_{\alpha}= \begin{cases}y_{\alpha} & \text { if } \alpha \in A, \\ \pi(p) & \text { if } \alpha \notin A .\end{cases}
$$

We clearly have $\pi_{A} \circ i=\operatorname{id}_{X^{A}}$, so it follows that if $f=\pi_{A} \circ h \circ i$ then $f$ is a homeomorphism. Further note that $f\left(p_{A}\right)=w_{A}$. We will define another homeomorphism $g: X^{\kappa} \rightarrow X^{A}$. First we define sets $B(q)$ similar to $A(q)$ for $q \in Q$ as follows:

$$
B(q)=\left\{\alpha \in A: w_{\alpha}=q\right\} .
$$

In fact we have $A(q)=B(q)$ if $q \neq q^{\prime}$ and $B\left(q^{\prime}\right)=A\left(q^{\prime}\right) \cup(A \backslash \kappa)$. It follows that $\left|B\left(q^{\prime}\right)\right|=\kappa=\left|A\left(q^{\prime}\right)\right|$. Fix a bijection $\xi: A\left(q^{\prime}\right) \rightarrow B\left(q^{\prime}\right)$. We define $g: X^{\kappa} \rightarrow X^{A}$ as follows for $y \in X^{\kappa}$ :

$$
g(y)_{\alpha}= \begin{cases}y_{\alpha} & \text { if } \alpha \notin B\left(q^{\prime}\right), \\ y_{\beta} & \text { if } \alpha \in B\left(q^{\prime}\right) \text { and } \beta=\xi^{-1}(\alpha) .\end{cases}
$$

One easily verifies that $g(x)=w_{A}$ and $g\left(p_{\kappa}\right)=p_{A}$. It follows that $g^{-1} \circ f \circ g$ is a homeomorphism of $X^{\kappa}$ that maps $p_{\kappa}$ onto $x$. This completes the proof.

Added in proof. The author has recently shown in [10] that Question 5.1 has a negative answer. This means that every $\Delta$-power homogeneous space is also power homogeneous.

\section{References}

[1] A. V. Arkhangel'skiǔ, Topological homogeneity. Topological groups and their continuous images, Uspekhi Mat. Nauk 42 (1987), no. 2 (254), 69-105, 287 (in Russian); English transl.: Russian Math. Surveys 42 (1987), no. 2, 83-131.

[2] A. V. Arkhangel'skiu, J. van Mill, and G. J. Ridderbos, A new bound on the cardinality of power homogeneous compacta, Houston J. Math., to appear.

[3] A. Bella, Remarks on the cardinality of a power homogeneous space, Comment. Math. Univ. Carolin. 46 (2005), 463-468.

[4] E. K. van Douwen, Nonhomogeneity of products of preimages and $\pi$-weight, Proc. Amer. Math. Soc. 69 (1978), 183-192.

[5] R. Engelking, General Topology, 2nd ed., Sigma Ser. Pure Math. 6, Heldermann, Berlin, 1989. 
[6] M. Ismail, Cardinal functions of homogeneous spaces and topological groups, Math. Japon. 26 (1981), 635-646.

[7] I. Juhász, Cardinal Functions in Topology - Ten Years Later, 2nd ed., Math. Centre Tracts 123, Mathematisch Centrum, Amsterdam, 1980.

[8] J. van Mill, On the cardinality of power homogeneous compacta, Topology Appl. 146/147 (2005), 421-428.

[9] G. J. Ridderbos, Notes on cardinal functions and homogeneity, Master Thesis, Vrije Univ., Amsterdam, 2004.

[10] - A characterization of power homogeneity, preprint.

[11] K. A. Ross and A. H. Stone, Products of separable spaces, Amer. Math. Monthly 71 (1964), 398-403.

[12] B. Šapirovskiı̌ [B. Shapirovskiū], Canonical sets and character. Density and weight in bicompacta, Dokl. Akad. Nauk SSSR 218 (1974), 58-61 (in Russian); English transl.: Soviet Math. Dokl. 15 (1974), 1282-1287.

[13] R. de la Vega, Homogeneity properties on compact spaces, Doctoral Thesis, Univ. of Wisconsin-Madison, 2005.

Faculty of Sciences, Division of Mathematics

Vrije Universiteit

De Boelelaan $1081^{\mathrm{A}}$

1081 HV Amsterdam, The Netherlands

E-mail: gfridder@few.vu.nl

URL: http://www.math.vu.nl/ gfridder

Received 16 September 2005;

in revised form 27 April 2006 